\title{
Interactive comment on "Dispersion in deep polar firn driven by synoptic-scale surface pressure variability" by Christo Buizert and Jeffrey P. Severinghaus
}

\section{Trudinger (Referee) \\ cathy.trudinger@csiro.au}

Received and published: 19 July 2016

This paper makes a convincing case for the existence, cause and magnitude of dispersion in deep polar firn. Dispersion in deep firn is an unresolved issue that needed further study, and this paper meets that need very well. It is well written and I recommend publication after consideration of the following comments.

Specific comments:

1) Page 1, line 27 (Fig 1): Please clarify whether the molecular diffusivity for the 'no LIZ mixing' case shown in blue was tuned to the available observations, or simply the same as the other case but run without any LIZ mixing? 
2) Page 2, line 28-30: I think it is worth being clearer about the distinction between the requirement for some diffusion in the LIZ, and whether that diffusion is most likely to be eddy-type rather than molecular. Not all of the models in Buizert et al (2012) used LIZ eddy-type diffusive mixing, but all did require some kind of diffusion in the LIZ. Therefore I think the case for some diffusion in the LIZ at NEEM is clear, but you need to take more care with the argument that the LIZ diffusion is eddy-type. The continued increase in d15N2 that would be associated with molecular diffusion is used as the reason for the LIZ diffusion being eddy not molecular, but how large is the increase in $\mathrm{d} 15 \mathrm{~N} 2$ that would be caused by the required amount of purely molecular diffusion in the LIZ? In Trudinger et al (2013, Supplementary Section 7), molecular diffusion in the LIZ in the CSIRO model that best matched observations of all 10 tracers at NEEM caused only a very small increase in $\mathrm{d} 15 \mathrm{~N}$ that appeared not to be inconsistent with the measurements. The two models in Buizert et al (2012) (LGGE-GIPSA and CSIRO) that didn't use LIZ eddy-type diffusion, but had LIZ molecular diffusion, perhaps did not match the $14 \mathrm{CO} 2$ peak quite as well as the models with LIZ eddy-type diffusion, but their agreement with measured 14CO2 (Fig 3e of Buizert et al., 2012) was much better than that shown by the blue line in Fig 1 of the present study (for no LIZ diffusion). The model-observation difference, RMSD, for the models in Buizert et al (2012) considering all 10 tracers is not significantly different for the cases with and without dispersion (taking the updated calculation for the CSIRO model presented in Trudinger et al., (2013) rather than the one given in Buizert et al (2012)). Specifically, the lowest RMSD is 0.73 for the CIC model (with dispersion) and the next lowest is 0.74 for the LGGE-GIPSA and CSIRO models without dispersion, so there is not a clear case for dispersion from the overall match with observations, and it is not true that all models 'require a LIZ eddy-type diffusive mixing' as stated at line 28 , just that they all required some type of LIZ diffusive mixing.

3) Page 9, line 2: I think that a clearer (but still brief) description of the experiment from Schwander et al (1988) should be given, more similar to the description in the original paper, e.g. "the small amount of $\mathrm{CO} 2$ or $\mathrm{O} 2$ was injected into the carrier gas". I'm not

Printer-friendly version

Discussion paper
Interactive comment 
sure what was meant by 'injected in front of the firn sample'. These measurements form an important part of the paper, so it would help the reader if you could provide a better description.

Technical corrections:

Page 9, Fig 5 caption, line 3: specify that this is the 95\% confidence interval.

Page 13, line 18: should be Fig 7a-b

Page 14, fig 8: the black text on dark blue contours is very hard to see, could some of the location names be written in white or another light color?

Page 15, line 1: Add 'of' after 'amount'

Interactive comment on The Cryosphere Discuss., doi:10.5194/tc-2016-148, 2016. 